\title{
Clinicopathological significance of KAl1 expression and epithelial-mesenchymal transition in non-small cell lung cancer
}

\author{
Lei Zhou ${ }^{\dagger}$, Lan Yư ${ }^{\dagger}$, Shiwu Wu ${ }^{* \dagger}$, Zhenzhong Feng, Wenqing Song and Xiaomeng Gong
}

\begin{abstract}
Background: KAl1 and epithelial-mesenchymal transition (EMT) is related to both angiogenesis and lymphangiogenesis and is an important target in new cancer treatment strategies. We aimed to investigate the KAl1 and marker of EMT expression and correlation with lymph node metastasis (LNM) and explore their prognostic impact in non-small cell lung cancer (NSCLC).

Methods: Tumor tissue specimens from 312 resected patients with stage I-IIIA NSCLC were obtained. Immunohistochemistry was used to assess the expression of the molecular markers KAl1, E-cadherin (E-cad), vimentin, CD34, and D2-40.

Results: There were $153 \mathrm{NO}$ and $159 \mathrm{~N}+$ patients. Tumor cell expression of KAl1and the marker of EMT, lymphatic vessel density (LVD), and microvessel density (MVD) were related to LNM. In multivariate analyses, the ages of patients, high tumor cell KAl1 expression, EMT, and the scores of MVD were independent factor of prognosis.

Conclusions: Tumor cell KAl1 expression, EMT, LVD, and MVD correlate with LNM. Thus, the detection of KAl1, expression of markers of EMT, and the scores of MVD may be used as a potential indicator of NSCLC prognosis.
\end{abstract}

Keywords: KAl1, Epithelial-mesenchymal transition, Angiogenesis, Lymphangiogenesis, NSCLC

\section{Background}

Lung cancer is the most frequent malignancy and the first leading cause of cancer-related death worldwide [1]. Complete surgical resection is the only potential curative treatment for localized lung cancer. About $65 \%$ non-small cell lung cancer (NSCLC) patients who are suitable for surgical procedures will relapse within 2 years and subsequently die of metastatic spread [2,3]. A common feature of tetraspanins is regulating cell motility. The mechanism that tetraspanins modulate cell motility is still unclear. KAI1 gene was originally identified as a suppressor of metastasis of tumor in prostate cancer and located on human chromosome 11p11.2 [4]. It is a member of the tetraspanins superfamily of glycoproteins. KAI1 suppresses metastasis by multiple mechanisms regulating inhibition of cell motility, adhesion, fusion, and proliferation [5]. It was

\footnotetext{
*Correspondence: 573448542@qq.com

${ }^{\dagger}$ Equal contributors

Department of Pathology, the First Hospital Affiliated of Bengbu Medical College, Bengbu Medical College, No. 287 Changhuai Ave, Longzihu, Bengbu, Anhui Province 233003, China
}

supposed that KAI1 exerted its function by modulating membrane structure by interactions with cell surface molecules, such as cell adhesion molecules and other tetraspanins. Many studies have shown that decreased KAI1 expression could be a useful marker for metastatic, invasive, and prognostic factor in many human tumors, such as lung [6], breast [7, 8], gastric [9, 10], liver [11], colorectal [12], bladder[13], esophageal [14], and prostatic cancer [15].

Epithelial-mesenchymal transition (EMT) is a reversible embryonic process and aberrantly activated in tumor progression and metastasis. Many studies have shown that EMT was a critical process in tumor invasion and metastasis in NSCLC $[16,17]$. It is commonly characterized by downregulation of E-cadherin (E-cad) which is a critical cell to cell adhesion molecule [18] and upregulation of vimentin which plays an important role in cell migration [19]. KAI1 may stabilize or strengthen E-cad-dependent cell-to-cell adhesion and motility by regulating $\beta$-catenin-mediated signal transduction on tumor cells, thus preventing tumor cells from seceding from the primary tumor [20]. These effects 
suggest that KAI1-induced E-cad signaling provides a survival benefit for metastatic tumors; however, whether KAI1 can inhibit EMT in NSCLC cells and the mechanism involved remains unclear.

\section{Methods}

\section{Patients and clinical samples}

Primary tumor tissues diagnosed NSCLC with pathologic stage I-IIIA from patients at the First Hospital Affiliated of Bengbu Medical College from 2003 to 2007 were used in this retrospective study. In total, 365 patients were registered from the hospital database. Of these, 53 patients were excluded from the study due to the following: (i) radiotherapy or chemotherapy before surgery $(n=15)$, (ii) other malignancy within 5 years before NSCLC diagnosis $(n=17)$, and (iii) inadequate paraffin-embedded fixed tissue blocks $(n=20)$. Thus, 312 patients with complete medical records and adequate paraffin-embedded tissue blocks were eligible. Due to financial or other reasons, 265 $(84.9 \%)$ patients were to postoperative therapy (postoperative routine chemotherapy or radiotherapy).

This report includes follow-up data as of 31 October 2012. The median follow-up was 42 (range 8-105) months. All specimens were obtained from the archives of the Department of Pathology at our hospital. The tumors were graded according to the World Health Organization and staged according to the International Union Against Cancer's tumor-node-metastasis classification. This study was approved by the ethical committee of Bengbu Medical College before it started.

\section{Immunohistochemistry}

All applied antibodies were subjected for immunohistochemistry (IHC) analysis on paraffin-embedded tissue. The antibodies used in this study were as follows: KAI1 (mouse monoclonal; Santa Cruz), E-cad (mouse monoclonal; LabVision), vimentin (mouse monoclonal; LabVision), CD34 (mouse monoclonal; LabVision), and D2-40 (mouse monoclonal; LabVision). The IHC procedures for these markers were previously described [21]. For each antibody, including negative controls, IHC was done in a single experiment.

\section{Scoring of IHC}

By light microscopy, representative tissue sections were scored semiquantitatively for cytoplasmic and membrane staining. All samples were anonymized and independently observed by two pathologists. If there is a disagreement, the observers would reexamine and reach a consensus. In scoring expression of antibodies, both the intensity and extent of immunopositivity were considered. The dominant staining intensity in tumors was scored as follows: $0=$ negative,
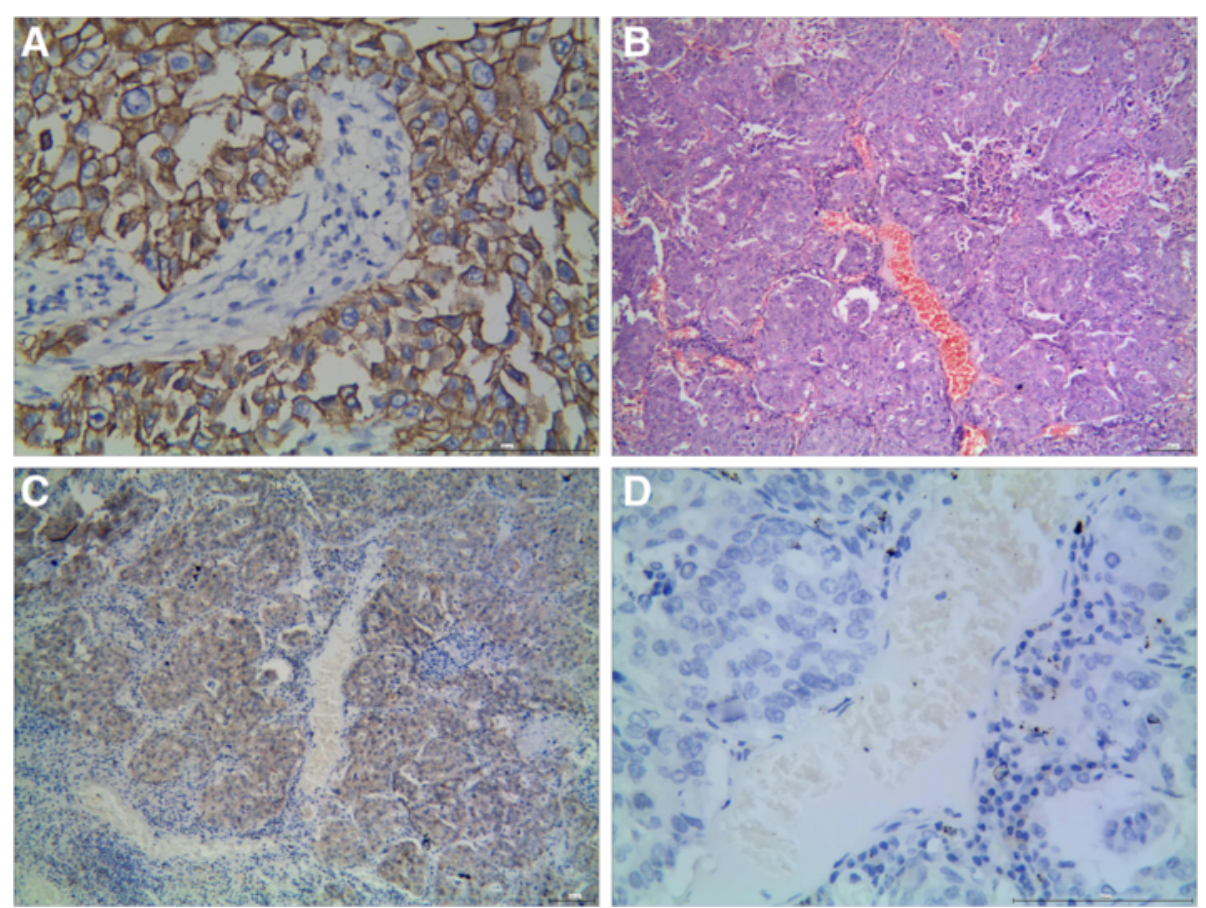

Fig. 1 Representative results of KAl1 and vimentin and E-cadherin protein in NSCLC. a Moderately differentiated NSCLC, H\&E staining. b KAl1 protein predominantly localized in the membrane and cytoplasm in well-differentiated squamous carcinoma. c NSCLC cells did not express a detectable level of E-cadherin protein. $\mathbf{d}$ Vimentin protein localized in the cytoplasm and membrane in NSCLC cells ( $\mathbf{a}, \mathbf{c}$, and $\mathbf{d}$ are serial sections) 
1 = weak, $2=$ moderate, and $3=$ strong. The extent of positive staining tumor cells was scored as follows: $<10 \%$ is $1,11-50 \%$ is $2,51-75 \%$ is 3 , and $>75 \%$ is 4 . The final score was determined by multiplying the intensity and the extent positivity scores, which yielded a range from 0 to 12 . Mean score from each individual was calculated in tumor cells. The positive expression for markers was scored as follows: $>2$, KAI1; $>3$, E-cadherin; and $>1$, vimentin.

The positive expression of KAI1 and E-cad was found mainly on the membrane and cytoplasm of NSCLC cells and normal lung tissues. The positive expression of vimentin was found mainly on the cytoplasm of NSCLC cells and normal lung tissues. They were presented as a brown granular material.

\section{Microvessel and lymphatic vessel density}

We assessed lymphatic vessel density (LVD) by D2-40 and microvessel density (MVD) by CD34 immunohistochemical staining. Not only stained endothelial cell but also endothelial cell cluster separated from other stromal elements was considered as a countable microvessel or lymphatic vessel.

\section{Statistical methods}

All statistical analyses were done using the statistical software SPSS (SPSS inc., Chicago, IL), version 17. The Fisher's exact test and Pearson chi-square test for trends in proportions, Spearman's correlate analysis, and Kaplan-Meier's method with log-rank test or Cox

Table 1 Correlation between KAl1 and vimentin and E-cad expression to clinicopathological characteristics in 312 NSCLC patients

\begin{tabular}{|c|c|c|c|c|c|c|c|c|c|}
\hline \multirow[t]{2}{*}{ Variable } & \multicolumn{2}{|l|}{ KAl1 } & \multirow{2}{*}{$\begin{array}{l}P \\
\text { value }\end{array}$} & \multicolumn{2}{|c|}{ E-cadherin } & \multirow{2}{*}{$\begin{array}{l}P \\
\text { value }\end{array}$} & \multicolumn{2}{|c|}{ Vimentin } & \multirow{2}{*}{$\begin{array}{l}P \\
\text { value }\end{array}$} \\
\hline & $(-)$ & $(+)$ & & $(-)$ & $(+)$ & & $(-)$ & $(+)$ & \\
\hline Ages & & & 0.622 & & & 0.894 & & & 0.336 \\
\hline$<60$ & 95 & 52 & & 88 & 59 & & 89 & 58 & \\
\hline$\geq 60$ & 111 & 54 & & 100 & 65 & & 91 & 74 & \\
\hline Gender & & & 0.038 & & & 0.423 & & & 0.597 \\
\hline Male & 147 & 87 & & 138 & 96 & & 137 & 97 & \\
\hline Female & 59 & 19 & & 50 & 28 & & 43 & 35 & \\
\hline Diameter & & & 0.081 & & & 0.070 & & & 0.368 \\
\hline$<3.0 \mathrm{~cm}$ & 32 & 9 & & 30 & 11 & & 21 & 20 & \\
\hline$\geq 3.0 \mathrm{~cm}$ & 174 & 97 & & 158 & 113 & & 159 & 112 & \\
\hline Histology & & & 0.283 & & & 0.077 & & & 0.323 \\
\hline SCC & 155 & 79 & & 144 & 90 & & 137 & 97 & \\
\hline Adenocarcinoma & 43 & 26 & & 36 & 33 & & 40 & 29 & \\
\hline LCC & 8 & 1 & & 8 & 1 & & 3 & 6 & \\
\hline Smoking & & & 0.957 & & & 0.791 & & & 0.185 \\
\hline Yes & 151 & 78 & & 139 & 90 & & 127 & 102 & \\
\hline No & 55 & 28 & & 49 & 34 & & 53 & 30 & \\
\hline Grades of tumor & & & $<0.001$ & & & $<0.001$ & & & 0.002 \\
\hline Well & 10 & 31 & & 13 & 28 & & 32 & 9 & \\
\hline Moderately & 135 & 68 & & 116 & 87 & & 118 & 85 & \\
\hline Poorly & 61 & 7 & & 59 & 9 & & 30 & 38 & \\
\hline TNM stages & & & $<0.001$ & & & $<0.001$ & & & $<0.001$ \\
\hline I & 10 & 57 & & 7 & 60 & & 65 & 2 & \\
\hline$\|$ & 85 & 35 & & 77 & 43 & & 89 & 31 & \\
\hline III & 111 & 14 & & 104 & 21 & & 26 & 99 & \\
\hline $\mathrm{Vl}$ & & & $<0.001$ & & & $<0.001$ & & & $<0.001$ \\
\hline Yes & 42 & 1 & & 40 & 3 & & 5 & 38 & \\
\hline No & 164 & 105 & & 148 & 121 & & 175 & 94 & \\
\hline Marginal free & & & 0.119 & & & 0.085 & & & $<0.001$ \\
\hline Yes & 171 & 95 & & 155 & 111 & & 167 & 99 & \\
\hline No & 35 & 11 & & 33 & 13 & & 13 & 33 & \\
\hline
\end{tabular}

NSCLC non-small cell lung cancer, SCC squamous cell carcinoma, LCC large cell carcinoma, VI vascular invasion 
Table 2 KAI1 and EMT and vascular endothelial and lymphatic endothelial marker as predictors for lymph node metastasis in 312 NSCLC patients $\left(X^{2}\right)$

\begin{tabular}{|c|c|c|c|c|c|c|c|}
\hline Marker expression & Patients ( $n$ ) & No $(n)$ & N1 $(n)$ & N2 $(n)$ & $\begin{array}{l}P\left(X^{2}\right) \text { No Verse N1 } \\
\text { Verse N2 }\end{array}$ & $\begin{array}{l}P\left(X^{2}\right) \mathrm{NO} \\
\text { Verse } \mathrm{N}+\end{array}$ & $\begin{array}{l}P\left(X^{2}\right) \mathrm{NO}+\mathrm{N} 1 \\
\text { Verse N2 }\end{array}$ \\
\hline KAl1 & & & & & $<0.001$ & $<0.001$ & $<0.001$ \\
\hline Negative & 206 & 84 & 80 & 42 & & & \\
\hline Positive & 106 & 69 & 34 & 3 & & & \\
\hline Co-expression & & & & & $<0.001$ & $<0.001$ & $<0.001$ \\
\hline Low V/high E & 114 & 75 & 36 & 3 & & & \\
\hline Low V/low E & 66 & 29 & 33 & 4 & & & \\
\hline High V/high E & 10 & 5 & 2 & 3 & & & \\
\hline High V/low E & 122 & 44 & 43 & 35 & & & \\
\hline MVD score & & & & & $<0.001$ & $<0.001$ & $<0.001$ \\
\hline Mean & $21.6 \pm 12.0$ & $18.0 \pm 11.5$ & $23.4 \pm 11.0$ & $29.2 \pm 11.2$ & & & \\
\hline LVD score & & & & & $<0.001$ & 0.005 & $<0.001$ \\
\hline Mean & $4.8 \pm 2.8$ & $4.4 \pm 2.7$ & $4.9 \pm 2.7$ & $6.2 \pm 2.5$ & & & \\
\hline
\end{tabular}

regression method for univariate or multivariate OS or disease-specific survival (DSS) analysis were used to assess the associations among the positive staining of KAI1, E-cad, vimentin, LVD, or MVD and clinicopathological indices. DSS was defined from the date of surgery to the time of NSCLC death. A value of $P<0.05$ was considered statistically significant.

\section{Results}

The relationship between expression of KAI1, E-cad, and vimentin and clinicopathological parameters

In NSCLC (Fig. 1b), the positive expression of KAI1 was $34.0 \%(106 / 312)$ (Fig. 1a), the positive expression of E-cad was $39.7 \%$ (124/312) (Fig. 1c), and the positive expression of vimentin was $42.3 \%(132 / 312)$ (Fig. 1d). There was a significant difference between the expression of KAI1, Ecad, and vimentin and grades of tumors $(P<0.001)$ and TNM stage $(P<0.001)$ (Table 1$)$.

\section{Expression of KAI1 and LNM and DSS (Table 2)}

The negative expression of KAI1 was seen significantly $(P<0.001)$ more often in $\mathrm{N}+(50.5 \%)$ than N0 when compared with positive expression of KAI1 (45.1\%).

The negative expression of KAI1 was seen significantly $(P<0.001)$ more frequent in N2 $(14.4 \%)$ than in

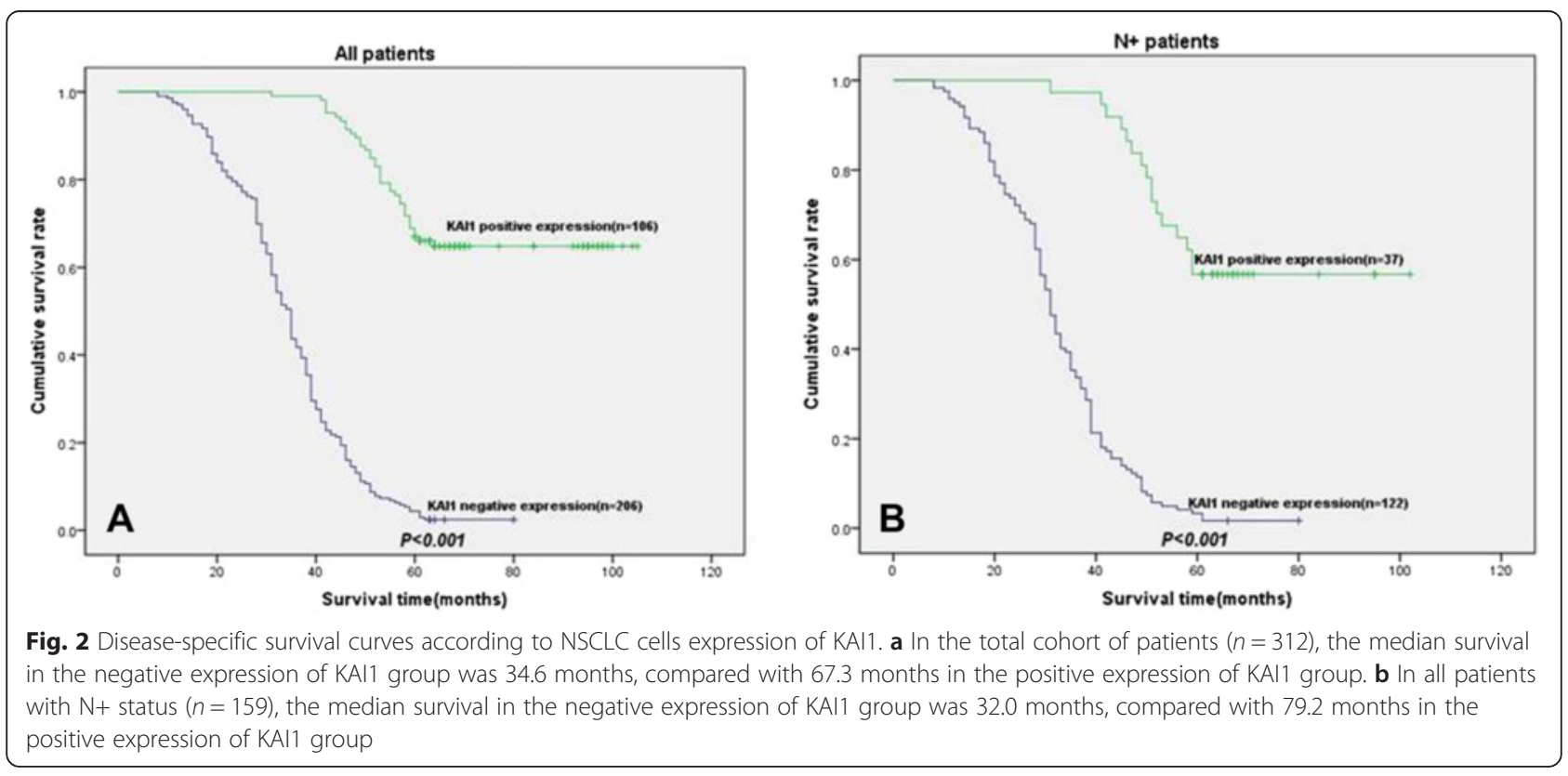




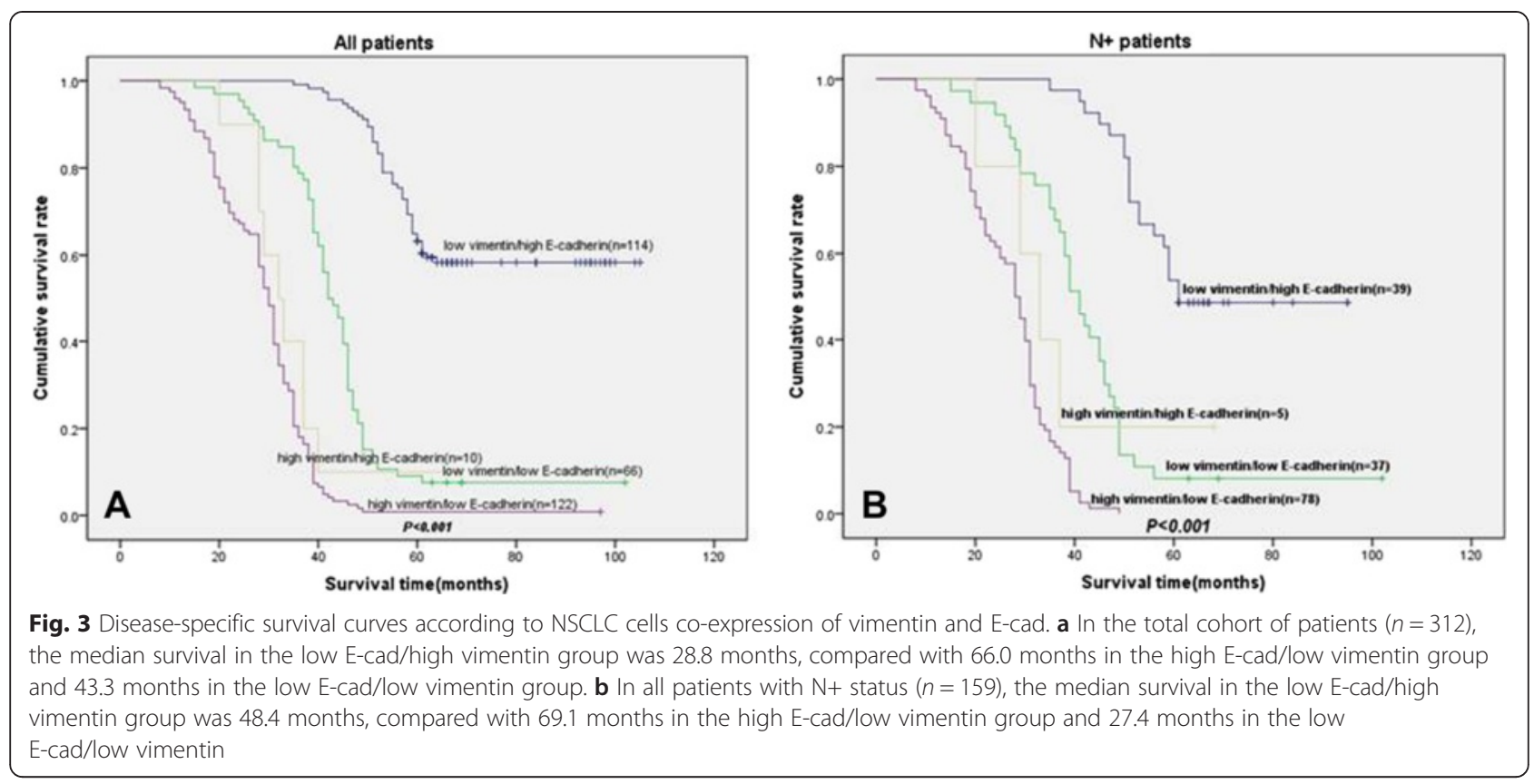

$\mathrm{N} 0+\mathrm{N} 1$ when compared with positive expression of KAI1 (38.6 \%).

In univariate analyses, the negative expression of KAI1 was clearly associated with poor survival $(P<0.001)$ as shown in Fig. 2a.

In the $\mathrm{N}+(\mathrm{N} 1+\mathrm{N} 2)$ patient cohort (159 patients), the negative expression of KAI1 group $(n=122)$ was related with a significantly worse prognosis $(P<0.001)$ when compared with the positive expression of KAI1 group (Fig. $2 \mathrm{~b}$ ).

\section{Co-expression of E-cad/vimentin and LNM (Table 2)}

Low E-cad/high vimentin expression was seen significantly $(P<0.001)$ more often in $\mathrm{N}+$ than $\mathrm{N} 0$ when compared with high E-cad/high vimentin (3.3\%), high E-cad/low vimentin (49.0\%), or low E-cad/low vimentin $(19.0 \%)$.

Low E-cad/high vimentin expression was seen significantly $(P<0.001)$ more frequent in $\mathrm{N} 2$ than in N0+N1 when compared with high E-cad/high vimentin (2.6\%),
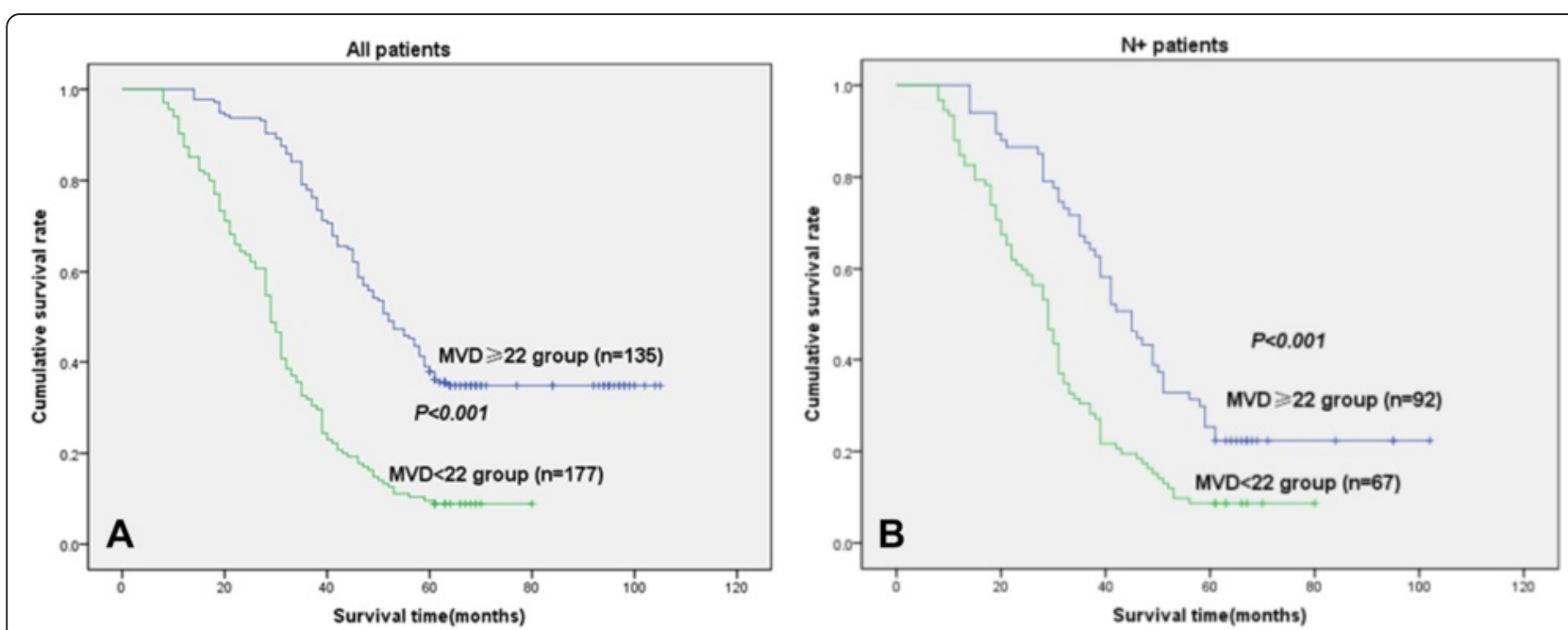

Fig. 4 Disease-specific survival curves according to NSCLC of MVD. a In the total cohort of patients $(n=312)$, the median survival in the MVD $<22$ group was 57.1 months, compared with 31.7 months in the MVD $\geq 22$ group. $\mathbf{b}$ In all patients with $\mathrm{N}+$ status $(n=159)$, the median survival in the $M V D<22$ group was 52.4 months, compared with 31.8 months in the MVD $\geq 22$ group (MVD $\geq 22$, because the mean score of MVD was 21.6 \pm 12.0 ) 


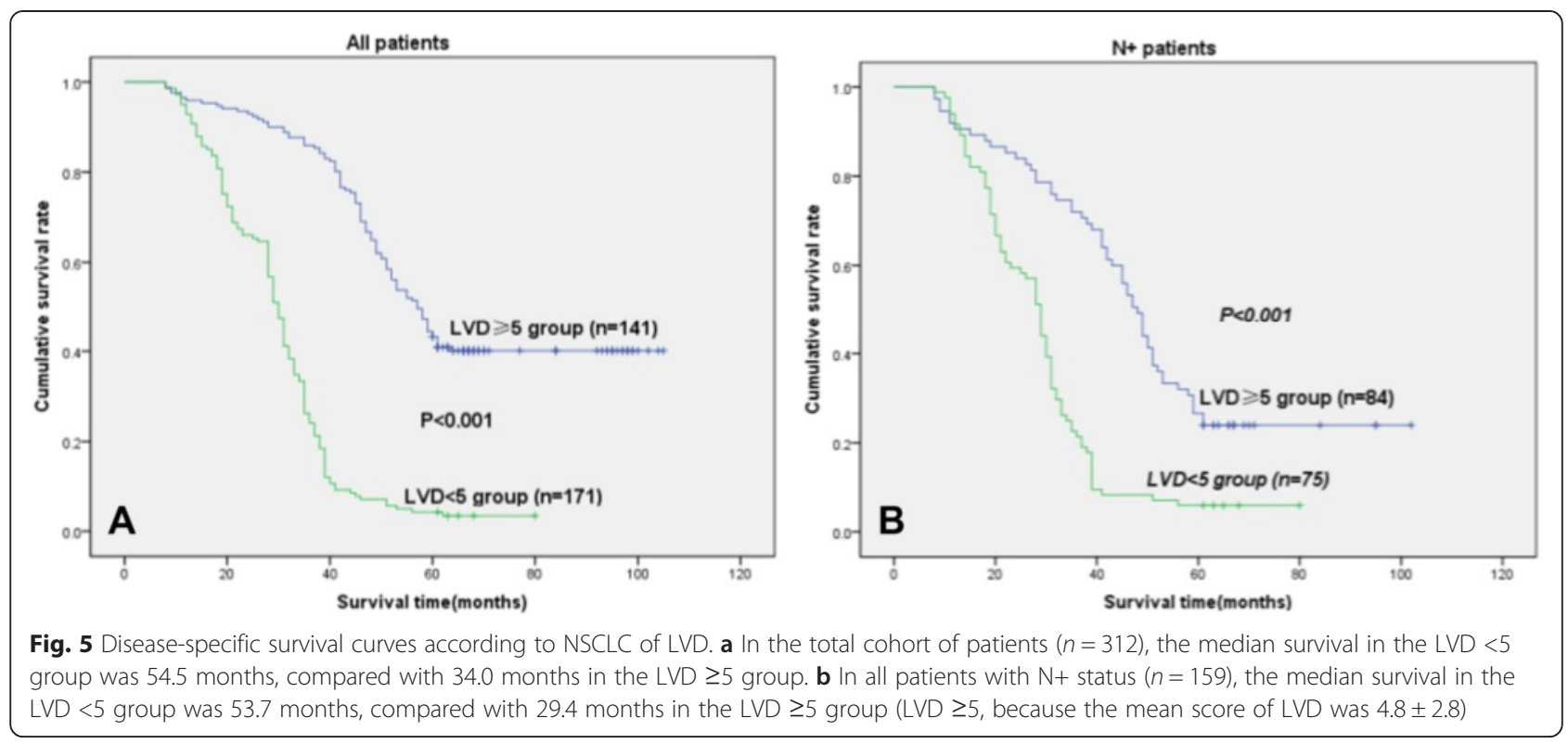

high E-cad/low vimentin (41.6\%), or low E-cad/low vimentin (23.2\%).

\section{Co-expression of E-cad/vimentin and DSS}

In univariate analyses, the co-expression of low E-cad/ high vimentin was clearly related with poor survival $(P<0.001)$ as shown in Fig. 3a.

In the $\mathrm{N}+(\mathrm{N} 1+\mathrm{N} 2)$ patient cohort (159 patients), the low E-cad/high vimentin expression group $(n=78)$ had a median survival of 26.7 months and was related with a significantly worse prognosis $(P<0.001)$ when compared with low E-cad/low vimentin and high E-cad/low vimentin expression group (Fig. 3b).

\section{LVD and MVD and LNM and DSS (Table 2)}

High scores of LVD $(5.2 \pm 2.7)$ and MVD $(25.0 \pm 11.4)$ were seen significantly $(P<0.01)$ more often in $\mathrm{N}+$ than N0 when compared with low scores of LVD $(4.4 \pm 2.7)$ and MVD $(18.0 \pm 11.5)$.

High scores of LVD $(6.2 \pm 2.5)$ and MVD $(29.2 \pm 11.2)$ were seen significantly $(P<0.001)$ more frequent in $\mathrm{N} 2$ than N0 + N1 when compared with low scores of LVD (4.6 \pm 2.7$)$ and MVD (20.3 \pm 11.6$)$.

In univariate analyses, the MVD $\geq 22$ group or LVD $\geq 5$ group was clearly associated with poor survival $(P<0.001)$ as shown in Fig. 4a or in Fig. 5a.

In the $\mathrm{N}+(\mathrm{N} 1+\mathrm{N} 2)$ patient cohort (159 patients), the MVD $\geq 22$ group $(n=92)$ or LVD $\geq 5$ group $(n=84)$ was related with a significantly worse prognosis $(P<0.001)$ when compared with the MVD $<22$ group $(n=67)$ or LVD $<5$ group $(n=75)$ (Fig. 4 b or Fig. $5 b)$.

In the multivariate Cox regression analysis, including all significantly clinicopathological parameters
(Table 1) and the co-expression E-cad/vimentin and the scores of LVD and MVD, the co-expression of Ecad/vimentin, the expression of KAI1, the score of MVD, pTNM stages, age of patients, marginal, and therapy emerged as independent prognostic factors for DSS (Table 3).

\section{Discussion}

In this study, we analyze 312 samples of surgically resected NSCLC Chinese patients using immunohistochemical method. We found that tumor cells expressed KAI1, E-cadherin, and vimentin protein to be independently associated with nodal metastasis and the coexpression of E-cadherin and vimentin and expression of KAI1 in tumor cells to be significantly associated with NSCLC patient prognosis.

To identify the relationship between KAI1 and EMT in NSCLC, five frequent indicator markers were detected in NSCLC tissues using IHC method. Many

Table 3 Multivariate survival analysis of 312 patients with NSCLC

\begin{tabular}{llllll}
\hline Covariate & $\mathrm{B}$ & $\mathrm{SE}$ & $\mathrm{Sig}$ & $\operatorname{Exp}(\mathrm{B})$ & $95 \% \mathrm{Cl}$ \\
\hline Age & -0.239 & 0.118 & 0.044 & 0.788 & $0.625-0.993$ \\
TNM stages & 0.311 & 0.21 & 0.010 & 1.365 & $1.078-1.730$ \\
KAl1 & -1.495 & 0.199 & $<0.001$ & 0.224 & $0.152-0.332$ \\
MVD score & 0.326 & 0.162 & 0.044 & 1.385 & $1.009-1.901$ \\
Marginal & 1.091 & 0.176 & $<0.001$ & 2.978 & $2.110-4.203$ \\
Co-expression & 0.633 & 0.074 & $<0.001$ & 1.882 & $1.628-2.176$ \\
Therapy & -0.551 & 0.170 & 0.001 & 0.576 & $0.413-0.804$ \\
\hline
\end{tabular}


studies [7, 20, 22-24] have indicated that KAI1 could regulate E-cad-dependent cellular adhesion in many tumors. In this study, we found that KAI1 protein expression was decreased in NSCLC and was correlated with grade of tumors and a poor prognosis, and so was Ecad. There was a positive relationship between the expression of KAI1 and E-cad. The low expression of KAI1 and E-cad lost their function which inhibited tumor cells invasion. In vivo data suggested that KAI1 be involved in EMT of NSCLC. We also found that there was a negative correlation between KAI1 and vimentin. These indicated that there was a potential role of KAI1 in EMT of NSCLC. However, to our knowledge, there was no study available on the association between the expression of KAI1 and EMT. The mechanism might have been the hypothesis as follows: Cell adhesion and motility, the functions of E-cad, were commonly regulated by $\beta$-catenin which forms $E$-cad$\beta$-catenin complexes. KAI1 could inhibit $\beta$-catenin tyrosine phosphorylation and stabilize E-cad- $\beta$-catenin complexes and thus prevent tumor cells dissemination from primary tumors [20]. The protein tyrosine kinase Src had been reported to be potent induces of EMT [25]. KAI1 expression could inhibit activation of Src kinase in the process of tumor malignancy and angiogenesis [26-28].

EMT has been found to be critical in tumor dissemination, local invasion, and metastasis, endowing cells with cancer stem cell properties [29-31]. Its common characteristic is downregulation of E-cad expression and upregulation of vimentin expression; this leads to numerous phenotypic changes such as the loss of cellular adhesion and polarity and the acquisition of migratory and invasive phenotype [32]. In this study, we have found strong diffuse expression of vimentin by immunohistochemistry and concomitant loss of E-cad expression in the majority of poorly differentiated NSCLC but not in normal lung tissue and well-differentiated NSCLC. We have found that the level of co-expression of E-cad/vimentin was associated with lymph node metastasis (LNM) and poor pathological TNM stages and poor clinical survival. This could be explained by the frequency invasive subpopulations of tumor cells resulting from EMT. Decreased E-cad and increased vimentin expression in LNM correlated with a shorter DSS. Many studies have shown that EMT phenomenon indicated a short survival in many human tumors [16, 17, 33-39]. But some authors did not believe that vimentin was associated with survival in NSCLC [16].

We observed a correlation of KAI1 and E-cad protein downregulation with vessel infiltration and metastasis in NSCLC. In contrast, vimentin protein is upregulated in NSCLC. These indicated that KAI1 and EMT should be involved in tumor cell invasion and metastasis. On the whole sections, we found that the strong KAI1 and E- cadherin expression of tumor cells was far from stromal cells. Upregulation expression of vimentin in tumor cells was often close to and inside intratumoral vessels. At the same time, the level of KAI1 and E-cad expression, less than normal lung tissues, was associated with low or absent vimentin expression in the majority of well or moderately differentiated tumors. But in poorly differentiated tumors, weak and absent E-cad expression was associated with the predominated expression of cytoplasmic vimentin. This study also found that high expression of KAI1 and E-cad could inhibit tumor angiogenesis and lymphangiogenesis. Increased vimentin expression could promote tumor angiogenesis and lymphangiogenesis. KAI1 inhibited $\beta$-catenin tyrosine phosphorylation, and this would stabilize the E-cad functions [20]. These findings might indicate that KAIl functions as a metastasis suppressor in the process of tumor invasion, angiogenesis, and lymphangiogenesis through inhibition of $\beta$-cateninmediated EMT.

\section{Conclusions}

It is suggested that KAI1 may play an important role in the LNM of NSCLC. Our results also show that KAI1 and EMT may be possible as a therapeutic marker for anti-metastasis therapy.

\section{Abbreviations}

NSCLC: non-small cell lung cancer; EMT: epithelial-mesenchymal transition; MVD: microvessel density; LNM: Iymph node metastasis; VI: vascular invasion; DSS: disease-specific survival; LVD: lymphatic vessel density; OS: overall; pTNM: pathological tumor-node-metastasis; IHC: immunohistochemistry; E-cad: E-cadherin.

\section{Competing interests}

The authors declare that they have no competing interests.

\section{Authors' contributions}

$L Z, L Y$, and SWW carried out the design, analysis of pathology, and drafted the manuscript. ZZF and WQS carried out sample collections and

coordination. XMG performed the immunohistochemical staining. All authors read and approved the manuscript.

\section{Acknowledgment}

This work was supported by Anhui Province Key Laboratory and Engineering Center (Bengbu Medical College) open issue plan (no. BYKL1412ZD) and the Natural Science key program of College and University of Anhui Province (No.KJ2015A269).

Received: 24 March 2015 Accepted: 20 July 2015

Published online: 01 August 2015

\section{References}

1. Ramalingam SS, Owonikoko TK, Khuri FR. Lung cancer: new biological insights and recent therapeutic advances. CA Cancer J Clin. 2011;61:91-112.

2. Devesa SS, Blot WJ, Stone BJ, Miller BA, Tarone RE, Fraumeni Jr JF. Recent cancer trends in the United States. J Natl Cancer Inst. 1995;87:175-82.

3. Donnem T, Al-Saad S, Al-Shibli K, Delghandi MP, Persson M, Nilsen MN, et al. Inverse prognostic impact of angiogenic marker expression in tumor cells versus stromal cells in non small cell lung cancer. Clin Cancer Res. 2007;13:6649-57.

4. Dong JT, Lamb PW, Rinker-Schaeffer CW, Vukanovic J, Ichikawa T, Isaacs JT, et al. KAl1, a metastasis suppressor gene for prostate cancer on human chromosome 11p11.2. Science. 1995;268:884-6. 
5. Smith SC, Theodorescu D. Learning therapeutic lessons from metastasis suppressor proteins. Nat Rev Cancer. 2009;9:253-64.

6. Miyake M, Adachi M, Huang C, Higashiyama M, Kodama K, Taki T. A novel molecular staging protocol for non-small cell lung cancer. Oncogene. 1999;18:2397-404.

7. Huang H, Groth J, Sossey-Alaoui K, Hawthorn L, Beall S, Geradts J. Aberrant expression of novel and previously described cell membrane markers in human breast cancer cell lines and tumors. Clin Cancer Res. 2005;11:4357-64.

8. Christgen $M$, Bruchhardt $H$, Ballmaier $M$, Krech $T$, Länger $F$, Kreipe $H$, et al. $\mathrm{KAl1/CD82}$ is a novel target of estrogen receptor-mediated gene repression and downregulated in primary human breast cancer. Int J Cancer. 2008;123:2239-46.

9. Chen Z, Gu S, Trojanowicz B, Liu N, Zhu G, Dralle H, et al. Downregulation of TM4SF is associated with the metastatic potential of gastric carcinoma TM4SF members in gastric carcinoma. World J Surg Oncol. 2011;9:43

10. Guan-Zhen Y, Ying C, Can-Rong N, Guo-Dong W, Jian-Xin Q, Jie-Jun W. Reduced protein expression of metastasis-related genes (nm23, KISS1, KAI1 and p53) in lymph node and liver metastases of gastric cancer. Int J Exp Pathol. 2007:88:175-83.

11. Guo XZ, Friess H, Di Mola FF, Heinicke JM, Abou-Shady M, Graber HU, et al. KAl1, a new metastasis suppressor gene, is reduced in metastatic hepatocellular carcinoma. Hepatology. 1998;28:1481-8.

12. Hashida H, Takabayashi A, Tokuhara T, Taki T, Kondo K, Kohno N, et al. Integrin alpha3 expression as a prognostic factor in colon cancer: association with MRP-1/CD9 and KAl1/CD82. Int J Cancer. 2002;97:518-25.

13. Jackson P, Rowe A, Grimm MO. An alternatively spliced KAl1 mRNA is expressed at low levels in human bladder cancers and bladder cancer cell lines and is not associated with invasive behaviour. Oncol Rep. 2007;18:1357-63.

14. Miyazaki T, Kato H, Shitara Y, Yoshikawa M, Tajima K, Masuda N, et al. Mutation and expression of the metastasis suppressor gene KAl1 in esophageal squamous cell carcinoma. Cancer. 2000;89:955-62.

15. Ueda T, Ichikawa T, Tamaru J, Mikata A, Akakura K, Akimoto S, et al. Expression of the KAl1 protein in benign prostatic hyperplasia and prostate cancer. Am J Pathol. 1996;149:1435-40.

16. Soltermann A, Tischler V, Arbogast S, Braun J, Probst-Hensch N, Weder W, et al. Prognostic significance of epithelial-mesenchymal and mesenchymal-epithelial transition protein expression in non-small cell lung cancer. Clin Cancer Res. 2008;14:7430-7.

17. Li LP, Lu CH, Chen ZP, Ge F, Wang T, Wang W, et al. Subcellular proteomics revealed the epithelial-mesenchymal transition phenotype in lung cancer. Proteomics. 2011;11:429-39.

18. Creighton CJ, Chang JC, Rosen JM. Epithelial-mesenchymal transition (EMT) in tumor-initiating cells and its clinical implications in breast cancer. J Mammary Gland Biol Neoplasia. 2010;15:253-60.

19. Kang Y, Massagué J. Epithelial-mesenchymal transitions: twist in development and metastasis. Cell. 2004;118:277-9.

20. Abe M, Sugiura T, Takahashi M, Ishii K, Shimoda M, Shirasuna K. A novel function of CD82/KAl-1 on E-cadherin-mediated homophilic cellular adhesion of cancer cells. Cancer Lett. 2008;266:163-70.

21. Wu S, Yu L, Wang D, Zhou L, Cheng Z, Chai D, et al. Aberrant expression of CD133 in non-small cell lung cancer and its relationship to vasculogenic mimicry. BMC Cancer. 2012;12:535.

22. Lee JH, Seo YW, Park SR, Kim YJ, Kim KK. Expression of a splice variant of KAl1, a tumor metastasis suppressor gene, influences tumor invasion and progression. Cancer Res. 2003;63:7247-55.

23. Shiwu WU, Lan $Y$, Wenqing $S$, Lei $Z$, Yisheng $T$. Expression and clinical significance of CD82/KAl1 and E-cadherin in non-small cell lung cancer. Arch Iran Med. 2012;15:707-12.

24. Chairoungdua A, Smith DL, Pochard P, Hull M, Caplan MJ. Exosome release of $\beta$-catenin: a novel mechanism that antagonizes Wnt signaling. J Cell Biol. 2010;190:1079-91.

25. Irby RB, Yeatman TJ. Role of Src expression and activation in human cancer. Oncogene. 2000;19:5636-42.

26. Sridhar SC, Miranti CK. Tetraspanin KAl1/CD82 suppresses invasion by inhibiting integrin-dependent crosstalk with c-Met receptor and Src kinases. Oncogene. 2006;25:2367-78.

27. Todeschini AR, Dos Santos JN, Handa K, Hakomori SI. Ganglioside GM2tetraspanin CD82 complex inhibits met and its cross-talk with integrins, providing a basis for control of cell motility through glycosynapse. J Biol Chem. 2007;282:8123-33.

28. Park JJ, Jin YB, Lee YJ, Lee JS, Lee YS, Ko YG, et al. KAl1 suppresses HIF-1a and VEGF expression by blocking CDCP1-enhanced Src activation in prostate cancer. BMC Cancer. 2012;12:81.

29. Mani SA, Guo W, Liao MJ, Eaton EN, Ayyanan A, Zhou AY, et al. The epithelial-mesenchymal transition generates cells with properties of stem cells. Cell. 2008;133:704-15.

30. Polyak K, Weinberg RA. Transitions between epithelial and mesenchymal states: acquisition of malignant and stem cell traits. Nat Rev Cancer. 2009:9:265-73.

31. Markiewicz A, Ahrends T, Wełnicka-Jaśkiewicz M, Seroczyńska B, Skokowski J, Jaśkiewicz J, et al. Expression of epithelial to mesenchymal transition-related markers in lymph node metastases as a surrogate for primary tumor metastatic potential in breast cancer. J Transl Med. 2012;10:226.

32. Thiery JP, Acloque H, Huang RY, Nieto MA. Epithelial-mesenchymal transitions in development and disease. Cell. 2009:139:871-90.

33. Andreolas C, Kalogeropoulou M, Voulgari A, Pintzas A. Fra-1 regulates vimentin during Ha-RAS-induced epithelial mesenchymal transition in human colon carcinoma cells. Int J Cancer. 2008;122:1745-56.

34. Armstrong AJ, Marengo MS, Oltean S, Kemeny G, Bitting RL, Turnbull JD, et al. Circulating tumor cells from patients with advanced prostate and breast cancer display both epithelial and mesenchymal markers. Mol Cancer Res. 2011;9:997-1007.

35. Katz E, Dubois-Marshall S, Sims AH, Gautier P, Caldwell H, Meehan RR, et al. An in vitro model that recapitulates the epithelial to mesenchymal transition (EMT) in human breast cancer. PLoS One. 2011;6:e17083.

36. Wang G, Dong W, Shen H, Mu X, Li Z, Lin X, et al. A comparison of Twist and $\mathrm{E}$-cadherin protein expression in primary non-small-cell lung carcinoma and corresponding metastases. Eur J Cardiothorac Surg. 2011;39:1028-32.

37. Pirozzi G, Tirino V, Camerlingo R, Franco R, La Rocca A, Liguori E, et al. Epithelial to mesenchymal transition by TGF $\beta-1$ induction increases stemness characteristics in primary non small cell lung cancer cell line. PLoS One. 2011;6:e21548.

38. Chaw SY, Majeed AA, Dalley AJ, Chan A, Stein S, Farah CS. Epithelial to mesenchymal transition (EMT) biomarkers-E-cadherin, beta-catenin, APC and Vimentin -in oral squamous cell carcinogenesis and transformation. Oral Oncol. 2012;48:997-1006.

39. Feng J, Zhang X, Zhu H, Wang X, Ni S, Huang J. FoxQ1 overexpression influences poor prognosis in non-small cell lung cancer, associates with the phenomenon of EMT. PLoS One. 2012;7:e39937.

\section{Submit your next manuscript to BioMed Central and take full advantage of:}

- Convenient online submission

- Thorough peer review

- No space constraints or color figure charges

- Immediate publication on acceptance

- Inclusion in PubMed, CAS, Scopus and Google Scholar

- Research which is freely available for redistribution 